\title{
A Survey of Massive Star Clusters in the X-ray Emission from Spiral Galaxies
}

\section{E. M. Schlegel}

High Energy Astrophysics Division, Smithsonian Astrophysical Observatory, Cambridge MA, USA

email: eschlegel@cfa.harvard.edu

\begin{abstract}
The massive star clusters identified by S. Larsen are compared to the available Chandra observations of face-on spiral galaxies. In each galaxy, a few percent of the Larsen-identified clusters match X-ray-emitting point sources. An additional few match knots of emission in the diffuse emission. The cluster properties are examined to ascertain whether massive star clusters are X-ray sources.
\end{abstract}

